\title{
Informação tecnológica: mapeando documentos de patentes e organizações atuantes no desenvolvimento de instrumentação agropecuária
}

\author{
Information technology: mapping patent documents and organizations acting in the \\ development of agricultural instrumentation
}

\begin{abstract}
Nayara Cristini Bessi
Mestranda em Ciência Tecnologia e Sociedade pela Universidade Federal de São Carlos - UFSCar E-mail: nayara@nit.ufscar.br

Douglas Henrique Milanez

Doutorando em Ciência e Engenharia de Materiais pela Universidade Federal de São Carlos - UFSCar

E-mail: douglas@nit.ufscar.br

Roniberto Morato do Amaral

Doutor em Engenharia de Produção pela Universidade Federal de São Carlos - UFSCar Professor do Departamento de Ciência da Informação da Universidade Federal de São Carlos - UFSCar

E-mail: roniberto@nit.ufscar.br
\end{abstract}

\begin{abstract}
Leandro Innocentini Lopes de Faria
Doutor em Ciência da Informação pela Universidade Paul Cézanne Aix Marseille III e em Ciência e Engenharia de Materiais pela Universidade Federal de São Carlos - UFSCar. Professor do Departamento de Ciência da Informação da Universidade Federal de São Carlos e do Programa de Pós-graduação em Ciência Tecnologia e Sociedade da UFSCar.

E-mail: leandro@nit.ufscar.br
\end{abstract}

\section{Resumo}

A cadeia produtiva do agronegócio tem um papel preponderante para o desenvolvimento socioeconômico dos países, principalmente do Brasil. Neste contexto, o avanço tecnológico em instrumentação agropecuária tem afetado significativamente a produtividade destas atividades tornando o campo cada vez mais competitivo. Atualmente não há como discorrer sobre competitividade sem tratar de informações externas que permitam às organizações conhecerem e compararem seus níveis de desenvolvimento e excelência. Com o intuito de fornecer informações tecnológicas competitivas, o objetivo deste artigo foi mapear as principais organizações globais atuantes no desenvolvimento de tecnologias em instrumentação agropecuária, suas áreas tecnológicas de maior interesse, os principais usos de suas tecnologias e seus mercados de atuação por meio de indicadores bibliométricos. Para tanto, foram utilizados 399.585 documentos de patentes indexados na base de dados Derwent Innovations Index no período de 2007 a 2011. A elaboração dos indicadores aliou as funcionalidades da base de dados às de um software bibliométrico a fim de lidar com o grande volume de dados encontrado. Considerando os principais titulares das patentes mapeados, houve um equilíbrio entre universidades e empresas, já as áreas tecnológicas de interesse identificadas estavam relacionadas à colheita, segadura, análise e investigação de materiais. Em geral, os principais usos das patentes foram referentes aos processos de replantio, colheita, alimentação e pesca e os mercados de destaque foram a China e os Estados Unidos, seguido da Coréia do Sul e do Japão.

Palavras-chave: Agronegócio. Instrumentação. Patentes. Análise bibliométrica. Tomada de decisão. 


\begin{abstract}
The Brazillian agribusiness production chain has a leading role in the socioeconomic development of countries. In this context, the technological advances in instrumentation have significantly affected the agricultural productivity making the field more competitive. Currently, competitiveness is strongly linked to external information wherein organizations can follow and compare their levels of development and excellence. In order to provide strategic technological information, the aim of this study was to map the major global organizations active in instrumentation agriculture, the main technological areas, application and markets using bibliometric indicators. Thus, we used 399.585 patent documents indexed in the database Derwent Innovations Index from 2007 to 2011. The indicators were developed combining features from database and bibliometric software in order to handle the large volume of data. To the main patentees mapped, there was a balance between universities and companies and the key technology areas of interest were related to harvesting, mowing, analysis and materials research. The main uses of the patents were related to the processes of replanting, harvesting, food and fishing and the highlighted markets were China and the United States, followed by South Korea and Japan
\end{abstract}

Keywords: Agribusiness. Instrumentation. Patents. Bibliometric analysis. Decision Making.

\title{
Introdução
}

Marco (1999) afirma que presenciamos a Era da Inteligência, atualmente mais importante do que somente selecionar a informação correta, em meio a um grande fluxo informacional, é dela extrair conhecimento estratégico. As organizações não operam de forma isolada, mas sim, se inter-relacionam em rede, geram mais valor do que outras, recebem destaque frente as demais, se tornam alvo de diversos tipos de comparações. Dessa maneira, não há como discorrer sobre competitividade sem tratar de informações externas comparativas que permitam organizações conhecerem seus níveis de competitividade e excelência (FUNDAÇÃO NACIONAL DA QUALIDADE, 2008).

Frente ao cenário competitivo, a sobrevivência e o destaque se dão por meio de inovações constantes. A inovação tecnológica pode ser compreendida como a inserção de um novo produto ou processo no mercado (ORGANIZAÇÃO PARA COOPERAÇÃO E DESENVOLVIMENTO ECONÔMICO, 2005) e as organizações buscam, com o desenvolvimento tecnológico, promover e proporcionar o bem-estar social, o desenvolvimento econômico e o desenvolvimento científico. Desse modo, comparar o nível de desenvolvimento tecnológico das organizações em uma determinada área é um processo preponderante para procurar novas parcerias, monitorar o comportamento de concorrentes e atuais parceiros, verificar as tecnologias que vem sendo desenvolvidas, entre outras questões (PORTER; NEWMAN, 2004).

Em análises de aspectos relacionados ao desenvolvimento tecnológico, um tipo de informação específica utilizada é a informação tecnológica, que pode ser entendida como todo

InCID: R. Ci. Inf. e Doc., Ribeirão Preto, v. 4, n. 1, p. 107-128, jan./jun. 2013. 
tipo de informação sobre tecnologias de fabricação, projeto e gestão que favoreça a melhoria contínua da qualidade e a inovação do setor produtivo (FACULDADE DE CIÊNCIA DA INFORMAÇÃO, 2004). Essas análises podem ser elaboradas utilizando indicadores quantitativos que sintetizam grandes quantidades de informações e facilitam o processo de análise, como os indicadores bibliométricos.

O Brasil vem conquistando posições competitivas no mercado internacional em vários segmentos de base tecnológica como, por exemplo, a produção de commodities do setor agrícola (CAMPOS; VALADARES, [2008?]). Além disso, o agronegócio possui um papel preponderante para o desenvolvimento socioeconômico do país, de tal forma que se estabelece uma importância econômica intrínseca ao aumento da produtividade agrícola, originada pelo desenvolvimento e aplicação da instrumentação agropecuária ao longo da cadeia produtiva do agronegócio.

$\mathrm{Na}$ literatura há vários estudos que extraem e sintetizam informações tecnológicas utilizando indicadores com o intuito de fornecer informação de valor agregado, dentre eles: Barroso et al. (2009); Faria (2001); Dou et al. (1990); Martin e Faria (2002); Milanez (2011); Peret et al. (2007); Porter e Newman (2004). No entanto, especificamente dentro da temática do agronegócio poucos estudos foram conduzidos (ALVES et al., 2006; RAMOS, 2012). Em consequência, há uma lacuna que necessita ser preenchida em virtude da importância que o agronegócio, em especial o papel da instrumentação agropecuária, representa para o Brasil.

Visto a importância do agronegócio para o avanço econômico e social brasileiro e do uso da instrumentação para o aumento da produtividade na cadeia do agronegócio, este artigo tem como objetivo mapear as principais organizações que vem se destacando em âmbito global no patenteamento em instrumentação agropecuária, por meio de indicadores bibliométricos derivados de documentos de patente indexados em bases de dados internacionais. O intuito foi fornecer informação tecnológica estratégica e mapear as organizações de destaque do setor; suas principais áreas tecnológicas de interesse; os principais usos de suas patentes e os mercados em que atuam. As seções seguintes discorrem sobre:

a) Como os conceitos de agronegócio, instrumentação e instrumentação agropecuária podem ser compreendidos e a importância econômica intrínseca ao desenvolvimento tecnológico em instrumentação agropecuária; 
b) Questões gerais relacionadas a processos de recuperação e análise da informação tecnológica;

c) Estratégia metodológica utilizada na aplicação da pesquisa seguido dos resultados do mapeamento e informações sobre as principais organizações atuantes em instrumentação agropecuária em âmbito global.

\section{Agronegócio e instrumentação: definições e importância econômica do desenvolvimento tecnológico em instrumentação agropecuária}

Nos últimos 50 anos a fisionomia das propriedades rurais mudou consideravelmente, pois elas perderam sua característica de autossuficiência no abastecimento dos mercados, tornaram-se mais dependentes de insumos e serviços e enfrentam os desafios da internacionalização e globalização da economia (ARAÚJO, 2007). Dessa maneira, o conceito de agronegócio deixou de ser somente rural, agrícola e primário, e passou a agregar muitos serviços, máquinas, insumos externos às propriedades, infraestruturas e mercados diversos, sendo melhor compreendido como:

O conjunto de todas as operações e transações envolvidas desde a fabricação dos
insumos agropecuários, das operações de produção nas unidades agropecuárias, até
o processamento e distribuição e consumo dos produtos agropecuários 'in natura' ou
industrializados (RUFINO, 1999, p.16).

Corroborando com esta ideia, Pereira e Paula (2006) afirmam que as atuais empresas rurais não se limitam mais apenas à produção, mas sim, estão preocupadas com toda uma sequência de fatores e agentes os quais formam uma cadeia produtiva que inicia-se na produção e passa pelo processamento, distribuição, venda e consumo. Nesse cenário o nível de competitividade no segmento do agronegócio tem se tornado cada vez mais acirrado, sendo a inovação e o desenvolvimento tecnológico de processos e produtos vital para a sobrevivência e destaque das organizações. Este fator tem como causa e consequência investimentos cada vez maiores em inovações feitas por instituições governamentais no setor. Dessa maneira, é evidente que o desenvolvimento tecnológico de instrumentos aplicados à cadeia produtiva do agronegócio é importante para o aumento da produtividade, da receita, do lucro que por consequência corroboram com a sobrevivência e o sucesso das empresas rurais dentro desse novo contexto do agronegócio (VIAN; ANDRADE JÚNIOR, 2010).

InCID: R. Ci. Inf. e Doc., Ribeirão Preto, v. 4, n. 1, p. 107-128, jan./jun. 2013. 
O conceito de instrumentação é complexo de ser compreendido devido à existência díspare de definições. Na literatura instrumentação pode ser entendida tanto como ciência, tanto como um conjunto de instrumentos. De acordo com a Instrument Society of American e American National Standards Institute (1979), instrumentação é uma ciência que aplica e desenvolve técnicas para medir e controlar equipamentos e processos industriais. Por outro lado, para Ribeiro (2007), instrumentação é o conjunto de instrumentos e equipamentos que são utilizados para detectar, observar, medir, controlar, comunicar e processar dados e sinais. Já a Embrapa Instrumentação Agropecuária (2008, p.18) a compreende como sendo:

\footnotetext{
a utilização de técnicas e conhecimentos avançados, métodos, sistemas e instrumentos para o desenvolvimento de equipamentos, metodologias, processos, novos materiais, sensores, medidores, controladores, atuadores, transmissores e processadores de sinal e para geração de novos conhecimentos para essa área, nos níveis das ciências básica e aplicada.
}

A disparidade de definições sobre instrumentação dificulta definir ao certo o que seria instrumentação dentro da perspectiva agropecuária e, além disso, esse conceito na visão de Octaviano (1995) ainda não possui uma terminologia bem estruturada. Este fator evidencia a necessidade de maiores estudos terminológicos que permitam delimitar essa área. Dada à dificuldade de conceituação, no presente artigo, foi necessário compreender e analisar instrumentação agropecuária em um contexto amplo, como sendo: um conjunto de instrumentos e equipamentos auxiliares utilizados em todas as operações e transações de fabricação, produção, processamento, distribuição e consumo de insumos e produtos agropecuários, mais especificamente, em toda cadeia produtiva do agronegócio.

No Brasil, o setor agropecuário é um dos principais fatores que fornecem poderio econômico e vantagem competitiva ao país por ser responsável por $22 \%$ do seu PIB (PORTAL BRASIL, 2010). Dessa forma, o uso e o desenvolvimento da instrumentação agropecuária ao longo cadeia produtiva do agronegócio e o aumento da produtividade ocasionado por ela, por ser de importância econômica e competitiva para as empresas rurais, têm corroborado consequentemente com o crescimento econômico do setor e do país.

De acordo com Campos e Valadares ([2008?]) o Brasil vem conquistando posições competitivas no mercado internacional em vários segmentos de base tecnológica, principalmente na produção de commodities agrícolas. Por se tratarem de produtos que são passíveis de poucas modificações e diferenciações, elas necessitam de avanços tecnológicos constantes, sendo assim, seu processo produtivo é cada vez mais dependente de fontes de 
pesquisa, aprimoramentos e tecnologias, a fim de alcançar por meio de seu processo produtivo qualidade diferenciada a custos menores (ARAÚJO, 2007).

Em síntese, a importância econômica intrínseca ao aumento da produtividade agrícola originada pelo desenvolvimento e aplicação da instrumentação agropecuária na cadeia de produção do agronegócio acirra o interesse de instituições pelo desenvolvimento constante de novas tecnologias na busca pelo poderio econômico e sobrevivência, provocando aumento na competitividade tecnológica em segmentos do setor agropecuário brasileiro e também no mercado globalizado (SAUER, 1998).

\section{Informação tecnológica disponível em documentos de patentes: recuperação e análise}

Em meio a essa competitividade as patentes tornaram-se o principal instrumento de proteção e negociação de direitos e fonte de informação tecnológica. Uma patente é um título de propriedade temporária concedido pelo Estado para uma invenção tecnológica ou modelo de utilidade depositada por um titular. Para obter uma patente, o interessado deve depositar o seu pedido em escritórios nacionais de cada país que lhe é de interesse. Se os critérios de patenteabilidade (novidade, atividade inventiva e aplicação industrial) são cumpridos e se lhe é concedido a carta patente, é permitido ao titular o direito de impedir terceiros de reproduzir ou comercializar a tecnologia desenvolvida e protegida sem seu consentimento.

Em contrapartida ao direito recebido, este titular é obrigado a revelar detalhadamente todo o conteúdo técnico da matéria de sua invenção (INSTITUTO NACIONAL DA PROPRIEDADE INDUSTRIAL, [2012?]; ORGANIZAÇÃO PARA COOPERAÇÃO E DESENVOLVIMENTO ECONÔMICO, 2009), transformando o documento de patente em informação tecnológica disponível para consulta e novos desenvolvimentos (FERREIRA et al., 2009). Consequentemente, informações de alto valor agregado podem ser extraídas desses documentos para subsidiar a tomada de decisão, entre as quais se destacam: as mudanças tecnológicas em um ambiente competitivo, a posição de uma organização em comparação a outras dentro de um campo tecnológico específico; as mudanças nas estratégias de desenvolvimento tecnológico de competidores, a contribuição para o conhecimento dos campos tecnológicos mais promissores para alocação de recursos de $\mathrm{P} \& \mathrm{D}$, entre outras questões (ERNST, 2003).

InCID: R. Ci. Inf. e Doc., Ribeirão Preto, v. 4, n. 1, p. 107-128, jan./jun. 2013. 
Os principais mercados de uma tecnologia também podem ser avaliados analisando o escopo geográfico dos depósitos de patentes (DEBACKERE; LUWEL, 2004). Segundo a Organização para Cooperação e Desenvolvimento Econômico (2009) quando há grande interesse econômico na exploração comercial, o titular da patente pode optar por proteger sua invenção em diversos países. Neste caso, análises de escopo geográfico requerem o uso de bases de dados supranacionais (possuem informações de patentes de vários escritórios nacionais). Além dos principais mercados, também pode ser analisada as principais áreas tecnológicas de interesse das organizações utilizando a Classificação Internacional de Patentes (CIP), que descreve a invenção por meio de códigos com significados préestabelecidos, independente dos termos utilizados na escrita do documento (ORGANIZAÇÃO MUNDIAL DA PROPRIEDADE INTELECTUAL, 2012).

Quando o pedido de patente é feito e analisado, as invenções por eles representadas são classificadas de acordo com oito seções principais: A (necessidades humanas); B (operações de processamento e transporte); C (química e metalurgia); D (têxteis e papel); E (construções fixas); F (engenharia mecânica, iluminação e aquecimento, armas e fornos); G (física) e H (eletricidade). A partir destas seções, a classificação subdivide-se em outros quatro níveis hierárquicos, conforme exemplificado no Quadro 1.

Quadro 1 - Subdivisões da Classificação Internacional de Patentes - CIP

Classificação Internacional de Patentes - CIP: A01B 1/24

Seção A: Necessidades humanas;

Classe A01: Agricultura; Silvicultura; Pecuária; Caça; Captura em armadilhas; Pesca;

Subclasse A01B: Trabalho do solo em agricultura ou silvicultura; peças, detalhes ou acessórios de máquinas ou implementos agrícolas, em geral;

Grupo principal A01B 1/00: Implementos manuais;

Subgrupo A01B 1/24: Implementos manuais para tratamento de prados ou gramados.

Fonte: Instituto Nacional de Propriedade Industrial, [2012?]

Em geral, os documentos de patentes depositados estão disponibilizados online em bases de dados de escritórios nacionais ou em bases comerciais. Os escritórios nacionais procuram manter acesso gratuito aos registros bibliográficos dos documentos, andamento de processos e cópia digital do documento publicado e concedido de seu país. São exemplos desses tipos de base a Braspat, mantida pelo Instituto Nacional da Propriedade Intelectual (INPI) e as bases do Escritório Americano de Patentes e Marcas (USPTO, sigla em inglês), do Escritório de Patente do Japão (JPO, sigla em inglês) e do Escritório de Propriedade Intelectual da República Popular da China (SIPO, sigla em inglês). Por outro lado, bases de 
dados comerciais compilam as informações disponíveis nas bases de acesso aberto e oferecem uma série de funcionalidades, principalmente na busca e recuperação das informações dos documentos de patente. Dentre as bases comerciais existentes se destacam a Derwent Innovations Index (DII), a Micropatent de propriedade da Thomson Reuters, e a Questel Orbit.

Em meio a uma grande quantidade de documentos de patentes, disponibilizados pelas bases de dados, e a necessidade atual de respostas rápidas em ambientes competitivos, técnicas quantitativas que sintetizam informações em forma de indicadores têm sido empregadas para analisar tendências tecnológicas e mapear concorrentes. Entre estas técnicas destaca-se a bibliometria, que é empregada na elaboração de indicadores e tratamento de dados bibliográficos dos documentos (OKUBO, 1997). O conceito de bibliometria foi cunhado em 1969 por Pritchard e, segundo ele, é um processo de aplicação de métodos e modelos estatísticos em livros e outros meios de comunicação. Com o passar do tempo a bibliometria se tornou um termo genérico e amplo para realização de mensurações específicas e elaboração de indicadores, principalmente para avaliação da produção científica e tecnológica, através de dados de publicações científicas e de documentos de patente (OKUBO, 1997).

Segundo Quoniam et al. (1993), o uso da bibliometria em análise de patentes é bem difundido na literatura, sendo relevante quando sua aplicação utiliza grandes volumes de registros, de maneira que é recomendado o uso de softwares de mineração de textos e de dados. Um exemplo desses tipos de software é o VantagePoint (VP), que fornece facilidades e ferramentas que contribuem no tratamento, contagem bibliométrica e análise grandes quantidades de dados brutos obtidos das bases de dados (VANTAGEPOINT, 2013). Embora existam diversos softwares para bibliometria, algumas bases de dados, entre as quais se destaca a DII, disponibilizam ferramentas que possibilitam análises bibliométricas preliminares dos resultados de uma busca, fornecendo insights iniciais a tomadores de decisão. 


\section{Estratégia metodológica}

A presente pesquisa pode ser caracterizada de acordo com sua natureza, como sendo um estudo aplicado, em relação ao objetivo, exploratória e explicativa por mapear as organizações atuantes em uma área tecnológica específica, utilizando-se da literatura disponível em documentos de patentes, além de compreender o comportamento dessas e os resultados dos indicadores. A abordagem que conduziu a pesquisa foi a quali-quantitativa e a técnica de análise adotada foi a bibliometria.

\section{Procedimento geral para elaboração dos indicadores}

Os indicadores bibliométricos foram elaborados aliando funcionalidades fornecidas pela base de dados DII e pelo software VP (versão 5.0) para lidar com grande volume de dados encontrados. Utilizando uma expressão de busca elaborada para instrumentação agropecuária, mostrada pela Tabela 1, foram recuperados 399.585 registros bibliográficos de documentos de patente indexados na base, em um intervalo (timespan) que compreendeu os anos de 2007 a 2011. Devido à dificuldade de coleta e tratamento desta grande quantidade de dados, o mapeamento das principais organizações que mais patenteiam no assunto de interesse foi efetuada com auxílio da ferramenta analítica analyze results da base DII. Essa ferramenta permite a geração de rankings a partir de informações que constam nos registros bibliográficos, em especial os titulares das patentes.

Como a base de dados agrupa em códigos fixos as diferentes denominações dos titulares mais ativos mundialmente em termos de patenteamento (assignee codes), criou-se selecionando a opção assignee code na ferramenta analítica um ranking com os 500 principais titulares. Do ranking foram extraídos as dez principais organizações atuantes a nível global e também verificou-se o significado de cada código em outra ferramenta de acesso online, da Thomson Reuters, conhecida como Patent Code Lookup ${ }^{l}$. Por fim, os dados bibliográficos dos documentos de patente das dez principais organizações foram recuperados manualmente e analisados com auxílio do software VP. Foram extraídos dos documentos de patentes recuperados os códigos da CIP, o resumo de uso e os países de origem e de depósito para elaboração dos seguintes indicadores:

\footnotetext{
${ }^{1}$ Patent Code Look up. Disponível em: <http://ipscience.thomsonreuters.com/support/patents/dwpiref/reftools/companycodes/lookup/>
}

InCID: R. Ci. Inf. e Doc., Ribeirão Preto, v. 4, n. 1, p. 107-128, jan./jun. 2013. 
a) Principais áreas tecnológicas de interesse a partir das subclasses dos códigos CIP;

b) Principais usos de suas patentes;

c) Mercados em que atuam verificando os principais países de depósito e de origem das patentes.

\section{Procedimento de recuperação: elaboração e aplicação de uma estratégia de busca}

De modo geral, uma boa estratégia de busca de patentes é composta de códigos e/ou palavras (ORGANIZAÇÃO MUNDIAL DA PROPRIEDADE INTELECTUAL, 2012). Na recuperação dos dados, a estratégia de busca foi elaborada utilizando como base agrupamentos da Classificação Internacional de Patentes, em domínios e subdomínios tecnológicos (OBSERVATOIRE DES SCIENCES ET DES TECHNIQUES, 2008), somados a palavras relacionadas aos contextos agronegócio e instrumentação. O procedimento adotado na elaboração da estratégia de busca foi uma adaptação das recomendações dadas por Watts e Porter (1997) A Tabela 1 disponibiliza a estratégia utilizada na recuperação dos dados.

Tabela 1 - Estratégia de busca

\#1 IP=(A01H* OR A21D* OR A23B* OR A23C* OR A23D* OR A23F* OR A23G* OR A23J* OR A23K* OR A23L* OR C12C* OR C12F* OR C12G* OR C12H* OR C12J* OR C13D* OR C13F* OR C13J* OR C13K* OR A01B* OR A01C* OR A01D* OR A01F* OR A01G* OR A01J* OR A01K* OR A01L* OR A01M* OR A21B* OR A21C* OR A22* OR A23N* OR A23P* OR B02B* OR C12L* OR C13C* OR C13G* OR $\left.\mathrm{C} 13 \mathrm{H}^{*}\right)$

\#2 TS=(agricult* OR agroindustr* OR agribusiness OR ("crop production") OR tillage* OR husbandry OR ("agrifood") OR agrifood* OR agrochemic* OR agronomic* OR aquacultur* OR cultivate* OR farm* OR food* OR forestry OR fruit* OR vegetable* OR harvest* OR horticultur* OR irrigat* OR legume* OR pulverization* OR silvicultur* OR veterinar* OR sow* OR livestock* OR soil* OR seed*)

\#3 TS=(bean* OR citric* OR citru* OR grain* OR grape* OR melon* OR onion* OR orange* OR peanut* OR pepper* OR potato* OR rice* OR sugarcane* OR coffee* OR cotton* OR corn OR corns OR wheat* OR banana* OR lemon* OR apple* OR tomato* OR cassava* OR watermelon*OR cocoa*)

\#4 TS=(biocid* OR fertilizer* OR insecticid* OR fungicid* OR herbicid* OR molluscicid* OR nematicid* OR raticid* OR rodenticid* OR acaricid* OR pesticide*)

\#5 TS=(bees OR bee OR bovine* OR canine* OR cow* OR dog* OR equine* OR fish* OR horse* OR pork* OR swine* OR chicken* OR poult* OR buffaloe* OR caprine* OR goat* OR sheep*)

\#6 \#5 OR \#4 OR \#3 OR \#2 OR \#1

\#7 IP= (G02* OR* G03B* OR G03C* OR G03D* OR G03F* OR G03G* OR G03H* OR H01S* OR G01B* OR G01C* OR G01D* OR G01F* OR G01G* OR G01H* OR G01J* OR G01K* OR G01L* OR G01M* OR G01N* OR G01P* OR G01R* OR G01S* OR G01V* OR G01W* OR G04* OR G05B* OR G05D* OR G07* OR G08B* OR G08G* OR G09B* OR G09C* OR G09D* OR G12* OR A61B* OR A61C* OR A61D* OR A61F* OR A61G* OR A61H* OR A61J* OR A61L* OR A61M* OR A61N* OR G01T* OR G21* OR H05G* OR $\mathrm{H} 05 \mathrm{H}^{*}$ )

InCID: R. Ci. Inf. e Doc., Ribeirão Preto, v. 4, n. 1, p. 107-128, jan./jun. 2013. 

\#8 IP= (A47J* OR B01J* OR B65B* OR B65D* OR C12M* OR D01D* OR E04H* OR F25B* OR G06G* OR H01J*)
\#9 TS=(accelerator* OR adder* OR agitator* OR analyser* OR applicator* OR aspirator* OR barometer* OR battery* OR bioinstrumentation* OR (bio-reactor*) OR boiler* OR bottle* OR cabinet* OR cable* OR calliper* OR camera* OR capaciflector* OR capacitance* OR capacitor* OR catcher* OR catching OR circuitry* OR circulator* OR collector* OR colorimeter*)

\#10 TS=(compartment* OR compressor* OR container* OR controller* OR transmitter* OR converter* OR (converter-sensor*) OR conveyor* OR cooler* OR cropper* OR cutter* OR cytometer* OR degasser* OR dehydrator* OR dendrometer* OR densimeter* OR depository* OR detector* OR determinator* OR device* OR diffuser* OR digger* OR digitizer* OR discharger* OR displayer* OR distributor* OR ditcher* OR dripper* OR drive* OR dryer* OR duster* OR ejector* OR electrometer* OR emulator* OR equalizer* OR equator* OR equipment*)

\#11 TS=(exchanger* OR exerciser* OR extractor* OR feeder* OR feeler* OR filter* OR forager* OR fumigator* OR gathering OR goniometer* OR harvester* OR processor* OR reaper* OR holder* OR homogenizer* OR hydrometer* OR hydrophone* OR hygrometer* OR incubator* OR instrument* OR interferometer* OR interrogator* OR Ionizer* OR leafcutter* OR limiter* OR liquefier* OR localizer* OR machine* OR (machinereadable*) OR machinery OR (machine-specific*) OR manometer* OR (measure-controller*) OR measurer*)

\#12 TS=(mechanism* OR microcomputer* OR (micro-computer*) OR microcontroller* OR micropump* OR (micro-sprayer*) OR (mini-lysimeter*) OR moistener* OR monitor* OR motor* OR multivibrator* OR object* OR ombrometer* OR orchidometer* OR pipe* OR lanimeter* OR planter* OR pot* OR processor* OR propeller* OR protractor* OR provider* OR pulsator*)

\#13 TS=(pulveriser* OR pumper* OR puncher* OR pusher* OR pyrheliometer* OR reaper* OR reflector* OR retainer* OR saturator* OR saucer* OR scraper* OR sensor* OR sower* OR spectrophotometer* OR stroboscope* OR supporter* OR telescope* OR thermalizer* OR thermometer* OR thermosensor* OR tool* OR tractor* OR transmitter* OR receiver* OR transponder* OR truck* OR vibrator*)

\#14 \#13 OR \#12 OR \#11 OR \#10 OR \#9 OR \#8 OR \#7

\#15 \#14 AND \#6

Fonte: Autor

\section{Procedimento para obtenção do uso das tecnologias patenteadas}

O uso das tecnologias foi identificado por meio de palavras-chave extraídas do campo uso $^{2}$ dos resumos da patente através de um processamento de linguagem natural (NLP) do software VP. O campo uso dos resumos da patente é elaborado a partir das informações contidas no texto completo dos documentos de patente (THOMSON, 2007). Este procedimento gerou uma lista das palavras que estavam contidas em uma maior quantidade de registros e, para deixar os dados uniformes e evitar redundâncias, foi feita a agregação dos termos por meio da funcionalidade de limpeza de dados do VP, chamado list clean up, que se baseia em lógica de semelhança para agrupar palavras. Desse modo, foi possível categorizar os principais usos do conjunto das patentes de cada organização.

\footnotetext{
${ }^{2}$ A DII procura melhorar o título e o resumo das patentes buscando conteúdo no texto completo do pedido e adicionando informações nos respectivos campos mencionados, a fim de melhorar o entendimento do usuário da base.
}

InCID: R. Ci. Inf. e Doc., Ribeirão Preto, v. 4, n. 1, p. 107-128, jan./jun. 2013. 


\section{Resultados e discussão}

\section{Principais organizações atuantes em instrumentação agropecuária}

Pelo número de documentos recuperados e o total de patentes verificadas para o primeiro colocado no ranking, apresentado pelo Gráfico 1, observa-se que a área de instrumentação agropecuária é bastante difusa em termos de atuantes. Em parte, isso é consequência da expressão de busca utilizada para a recuperação dos dados, aliada a dificuldade de encontrar uma terminologia que defina bem a área.

\section{7-2011}

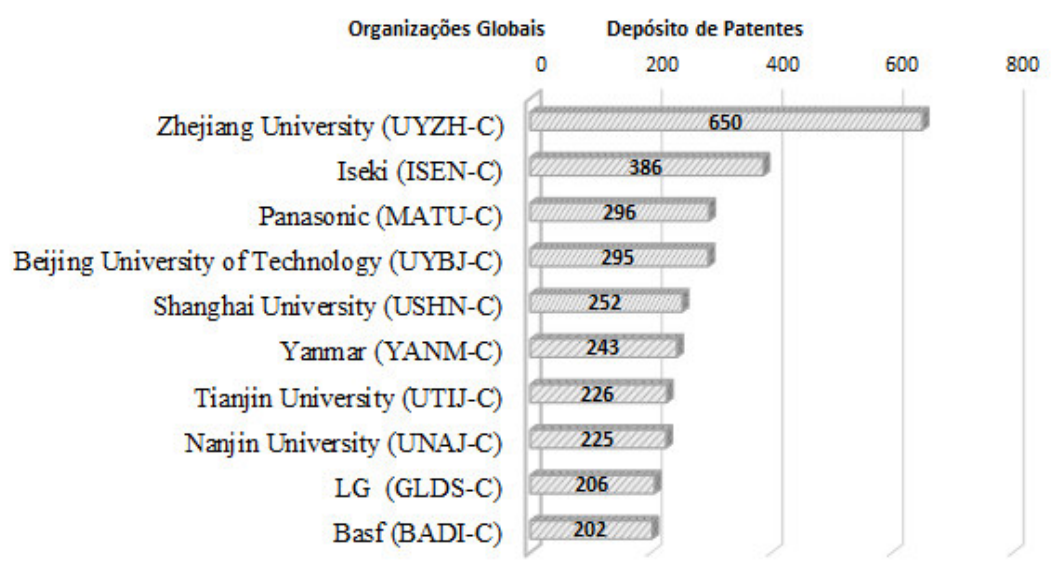

Gráfico 1 - Ranking das principais organizações globais em instrumentação agropecuária Fonte: Derwent Innovations Index. Elaborado pelos autores.

Considerando as 10 organizações listadas, nos últimos 4 anos houve equilíbrio entre os depósitos de universidades (50\%) e empresas (50\%), com destaque para a universidade chinesa Zhejiang University, responsável por somente 0,21\% das patentes recuperadas, seguida das empresas Iseki $(0,10 \%)$ e Panasonic $(0,07 \%)$ e da Beijing University of Technology $(0,07 \%)$, conforme Gráfico 1. Das universidades mapeadas todas são governamentais e chinesas e este fato pode ser consequência, pelo menos em parte, da reestrutução do sistema de propriedade intelectual que a China vivenciou no início deste século como requisito para fazer parte do Acordo Sobre Aspectos dos Direitos de Propriedade Intelectual Relacionados ao Comércio (TRIPS) e para a sua entrada na Organização Mundial do Comércio (OMC) em 2000 (HU; JEFFERSON, 2009; MILANEZ, 2011). Além disso, o aparecimento das cinco universidades públicas chinesas no ranking pode ser um indício, ou mesmo um reflexo, da preocupação do governo com o aumento da produção agropecuária por 
meio do desenvolvimento de novas tecnologias. De acordo com Nassar e Nogueira (2007), após a entrada da China na Organização Mundial do Comércio houve uma mudança estrutural também no seu setor agrícola, a estrutura tarifária das principais commodities foi fortemente reduzida, além de outras políticas domésticas que afetavam o setor. Desde 2004 o país vem buscando explorar suas vantagens competitivas como a grande quantidade de mão-de-obra e baixos custos, seguindo a estratégia de promover o desenvolvimento de produtos agrícolas intensivos de alto valor agregado, como frutas, vegetais e carnes, especialmente aves e suínos, estimulando desenvolvimento da indústria de processamento de alimentos como forma de gerar renda e dar competitividade ao produtor e ao país.

Em relação às empresas encontradas no Gráfico 1, três delas são de origem japonesa, uma é sul-coreana e uma é alemã. Tendo em vista o território japonês ser montanhoso, com várias regiões vulcânicas, e o país ser uma potência científica e tecnológica com mão-de-obra altamente capacitada e especializada, é provável que o destaque das empresas japonesas no desenvolvimento de instrumentos agropecuários venha de uma grande necessidade de utilização e desenvolvimento destes instrumentos que os auxiliem principalmente no trabalho deste solo montanhoso para o plantio, cultivo e criação de animais.

As empresas mapeadas podem ser divididas em dois grandes grupos de acordo com seus produtos e serviços relacionados: à agricultura e conservação e ao uso doméstico. No primeiro grupo podemos destacar as empresas japonesas Iseki, desenvolvedora e fornecedora de máquinas de plantio, colheita e de processamentos variados como: tratores, cultivadores, perfilhos, colheitadeiras, blinders, etc.; a Yanmar, desenvolvedora de produtos, dentre outros setores, para a agricultura como: tratores; micro tratores; roçadeiras; bombas de alta-pressão; derriçadeiras e pulverizadores; e a alemã Basf desenvolvedora de fungicidas, herbicidas, inseticidas e sementes. Em relação ao segundo grupo destacam-se a empresa japonesa Panasonic e a sul-coreana LG, desenvolvedoras de entre outras soluções, eletrodomésticos como refrigeradores e fornos para alimentos. Das cinco empresas mapeadas somente a Iseki não possui sede e atuação direta no Brasil (BASF, 2005; ISEKI, [entre 1997 e 2013]; LG ELECTRONICS, 2012; PANASONIC CORPORATION, 2013; YANMAR, 2010). 


\section{Áreas tecnológicas de interesse e principais usos das patentes das organizações}

Os Quadros 2 e 3 sintetizam os resultados encontrados referentes às áreas tecnológicas de interesse das organizações e dos usos de suas patentes. Verifica-se que tais atuações foram as relacionadas à colheita, segadura (A01D) e análise e investigação de materiais pela determinação de suas propriedades físicas e químicas (G01N). O uso das patentes de todas as organizações estão relacionados principalmente aos processos de replantio (174 registros); ao processo de colheita (104 registros); à alimentação e/alimentos (87 registros) e peixes e/ou pesca (67).

Quadro 2 - Principais áreas tecnológicas e principais usos das patentes das universidades (2007-2011)

\begin{tabular}{|c|c|c|}
\hline $\begin{array}{c}\text { Organizações } \\
\text { Globais }\end{array}$ & Principais áreas tecnológicas de interesse (Subclasse) & Principais usos \\
\hline $\begin{array}{l}\text { Zhejiang } \\
\text { University }\end{array}$ & $\begin{array}{l}\text { A01K }\left(\mathbf{7 8}^{3}\right) \text { - pecuária; tratamento de aves, peixes, insetos; piscicultura; } \\
\text { criação ou reprodução de animais; novas criações de animais. } \\
\text { A01C (68) - plantio; semeadura; fertilização. } \\
\text { G01N (67) - investigação ou análise dos materiais pela determinação de } \\
\text { suas propriedades químicas ou físicas. } \\
\text { A23L (52) - alimentos, produtos alimentícios ou bebidas não alcoólicas; } \\
\text { seu preparo ou tratamento. }\end{array}$ & $\begin{array}{c}\text { Replantio }\left(44^{4}\right) \\
\text { Alimentos (39) } \\
\text { Peixes e Pesca (40) } \\
\text { Plantio (27) }\end{array}$ \\
\hline $\begin{array}{c}\text { Beijing } \\
\text { University of } \\
\text { Technology }\end{array}$ & $\begin{array}{l}\text { G01N (30) - investigação ou análise dos materiais pela determinação de } \\
\text { suas propriedades químicas ou físicas } \\
\text { C02F (23) - tratamento de água, de águas residuais, de esgotos ou de } \\
\text { lamas e lodos } \\
\text { B01J (22) - processos químicos ou físicos, por ex., catálise, química } \\
\text { coloidal; aparelhos pertinentes aos mesmos } \\
\text { A01G (20) - horticultura; cultivo de vegetais, flores, arroz, frutas, vinhas, } \\
\text { lúpulos ou algas; silvicultura; irrigação }\end{array}$ & $\begin{array}{c}\text { Solo (35) } \\
\text { Plantas (17) } \\
\text { Campo (16) } \\
\text { Alimentos (13) }\end{array}$ \\
\hline $\begin{array}{l}\text { Shanghai } \\
\text { University }\end{array}$ & $\begin{array}{l}\text { A01K (50) - pecuária; tratamento de aves, peixes, insetos; piscicultura; } \\
\text { criação ou reprodução de animais, não incluídos em outro local; novas } \\
\text { criações de animais } \\
\text { G01N (26) - investigação ou análise dos materiais pela determinação de } \\
\text { suas propriedades químicas ou físicas } \\
\text { A01N (14) - Conservação de corpos de seres humanos ou animais ou } \\
\text { plantas ou partes dos mesmos; biocidas } \\
\text { A01P (14) - atividade de compostos químicos ou preparações biocidas, } \\
\text { repelentes ou atrativos de pestes ou reguladores do crescimento de plantas }\end{array}$ & $\begin{array}{c}\text { Peixes e Pesca (27) } \\
\text { Alimentos (16) } \\
\text { Teste (11) } \\
\text { Controle (10) }\end{array}$ \\
\hline $\begin{array}{c}\text { Tianjin } \\
\text { University }\end{array}$ & $\begin{array}{l}\text { A23L (31) - alimentos, produtos alimentícios ou bebidas não alcoólicas; } \\
\text { seu preparo ou tratamento } \\
\text { C12P (23) - processos de fermentação ou processos que utilizem enzimas } \\
\text { para sintetizar uma composição ou composto químico desejado ou para } \\
\text { separar isômeros ópticos de uma mistura racêmica } \\
\text { C12N (21) - micro-organismos ou enzimas; suas composições; } \\
\text { propagação, conservação, ou manutenção de micro-organismos; } \\
\text { engenharia genética ou de mutações; meios de cultura } \\
\text { B01J (20) -processos químicos ou físicos, catálise, química coloidal; } \\
\text { aparelhos pertinentes aos mesmos }\end{array}$ & $\begin{array}{c}\text { Alimentos (19) } \\
\text { Água (11) } \\
\text { Medicina (7) } \\
\text { Plantas (7) }\end{array}$ \\
\hline
\end{tabular}

\footnotetext{
${ }^{3}$ Quantidade de registros do ator que continham o código A01K, um registro pode ser classificado em mais de um código

${ }^{4}$ Quantidade de registros do ator que continham a palavra Replantio, um registro pode ser classificado em mais de uma palavra
}

InCID: R. Ci. Inf. e Doc., Ribeirão Preto, v. 4, n. 1, p. 107-128, jan./jun. 2013. 


\begin{tabular}{c|l|c}
\hline & G01N (32) - investigação ou análise dos materiais pela determinação de & \\
& suas propriedades químicas ou físicas & \\
& C12N (31) - micro-organismos ou enzimas; suas composições; & Teste e detecção (19) \\
Nanjin & propagação, conservação, ou manutenção de micro-organismos; & Solo (16) \\
engenharia genética ou de mutações; meios de cultura & Alimentos (13) \\
University & A61K (24) - preparações para finalidades médicas, odontológicas ou & Medicina (13) \\
& higiênicas & \\
& C02F (24) - tratamento de água, de águas residuais, de esgotos ou de & \\
& lamas e lodos & \\
\hline
\end{tabular}

Fonte: Derwent Innovations Index. Elaborado pelos autores.

Quadro 3 - Principais áreas tecnológicas e principais usos das patentes das empresas (20072011)

\begin{tabular}{|c|c|c|}
\hline $\begin{array}{c}\begin{array}{c}\text { Organizações } \\
\text { Globais }\end{array} \\
\end{array}$ & Principais áreas tecnológicas de interesse (Subclasse) & Principais usos \\
\hline Iseki & $\begin{array}{l}\text { A01D (133) - colheita; segadura } \\
\text { A01C (108) - plantio; semeadura; fertilização } \\
\text { A01F (47) - processamento do produto colhido; enfardamento de palha, } \\
\text { feno ou similares; aparelho estacionário ou instrumentos manuais para } \\
\text { formação ou enfeixamento de palha, feno ou similares em medas; corte de } \\
\text { palha, feno ou similares; armazenamento de produtos agrícolas ou } \\
\text { hortícolas } \\
\text { A01B (46) - trabalho do solo em agricultura ou silvicultura; peças, detalhes } \\
\text { ou acessórios de máquinas ou implementos agrícolas, em geral }\end{array}$ & $\begin{array}{l}\text { Replantio (84) } \\
\text { Colheita (62) } \\
\text { Controle (19) } \\
\text { Moagem (19) }\end{array}$ \\
\hline Panasonic & $\begin{array}{l}\text { A47J (81) - equipamento de cozinha; moedores de café; moedores de } \\
\text { especiarias; aparelhos para fazer bebidas } \\
\text { H01J (41) - válvulas de descarga elétrica ou lâmpadas de descarga } \\
\text { F25D (39) - refrigeradores; câmaras frigoríficas; geladeiras; aparelhos de } \\
\text { resfriamento ou congelamento } \\
\text { H05B (36) - aquecimento elétrico; iluminação elétrica }\end{array}$ & $\begin{array}{c}\text { Cozimento (94) } \\
\text { Aquecimento (65) } \\
\text { Refrigeração (47) } \\
\text { Armazenamento (15) }\end{array}$ \\
\hline Yanmar & $\begin{array}{l}\text { A01D (73) - colheita; segadura } \\
\text { A01C (41) - plantio; semeadura; fertilização } \\
\text { B60K (39) - disposições ou montagem de unidades de propulsão ou de } \\
\text { transmissões em veículos; disposição ou montagem de várias máquinas } \\
\text { motrizes diferentes; acionamentos auxiliares; instrumental ou painéis de } \\
\text { instrumentos para veículos; dispositivos correlatos a resfriamento, tomada } \\
\text { de ar, exaustão de gás ou alimentação de combustível de unidades de } \\
\text { propulsão, em veículos } \\
\text { A01F (38) - processamento do produto colhido; enfardamento de palha, } \\
\text { feno ou similares; aparelho estacionário ou instrumentos manuais para } \\
\text { formação ou enfeixamento de palha, feno ou similares em medas; corte de } \\
\text { palha, feno ou similares; armazenamento de produtos agrícolas ou } \\
\text { hortícolas }\end{array}$ & $\begin{array}{c}\text { Replantio (46) } \\
\text { Colheita (42) } \\
\text { Construção (22) } \\
\text { Geração (12) }\end{array}$ \\
\hline LG & $\begin{array}{l}\text { F25D (57) - refrigeradores; câmaras frigoríficas; geladeiras; aparelhos de } \\
\text { resfriamento ou congelamento } \\
\text { F24C (49) - outras estufas ou fogões domésticos; detalhes de estufas ou } \\
\text { fogões domésticos de aplicação geral } \\
\text { H01L (19) - dispositivos semicondutores; dispositivos elétricos de estado } \\
\text { sólidos não incluídos em outro local } \\
\text { A47J (17) - equipamento de cozinha; moedores de café; moedores de } \\
\text { especiarias; aparelhos para fazer bebidas }\end{array}$ & $\begin{array}{c}\text { Refrigeração (64) } \\
\text { Alimentos (58) } \\
\text { Cozimento (32) } \\
\text { Armazenamento (30) }\end{array}$ \\
\hline
\end{tabular}

InCID: R. Ci. Inf. e Doc., Ribeirão Preto, v. 4, n. 1, p. 107-128, jan./jun. 2013. 


\begin{tabular}{c|l|c}
\hline & A01N (80) - conservação de corpos de seres humanos ou animais ou & \\
& plantas ou partes dos mesmos; biocidas. & Controle (64) \\
A01P (56) - atividade de compostos químicos ou preparações biocidas, & Proteção (61) \\
Basf & repelentes ou atrativos de pestes ou reguladores do crescimento de plantas & Alimentos (36) \\
& C07D (47) - compostos heterocíclicos & Propagação (25) \\
& A61K (29) - preparações para finalidades médicas, odontológicas ou & \\
\hline
\end{tabular}

Fonte: Derwent Innovations Index. Elaborado pelos autores.

Evidencia-se no Quadro 3 que as empresas contribuíram totalmente (206 documentos) para o destaque área tecnológica relacionada à colheita e segadura, seguida da área de pecuária e tratamento de aves (128 documentos). O mesmo aconteceu com as universidades, como evidenciado no Quadro 2, porém a contribuição total (155 documentos) foi referente à área de análise e investigação de materiais pela determinação de suas propriedades físicas e químicas (155 registros), seguido pela área de plantio, semeadura e fertilização (149 documentos). Em relação ao uso das tecnologias patenteadas pelas universidades o destaque foi referente ao uso em alimentos (100 documentos) e peixes e pesca (67 documentos). Para as empresas, as tecnologias referiam-se ao replantio (130 documentos) e refrigeração (111 documentos).

\section{Principais mercados de interesse}

Conforme Quadro 4, observou-se que os principais mercados de interesse foram a China (211 documentos ${ }^{5}$ ) e os Estados Unidos (211 documentos), seguidos da Coréia do Sul (70 documentos) e do Japão (2 documentos), conforme apresentado no Quadro 4. As universidades chinesas se atentaram principalmente ao depósito em seus países de origem e, de um modo geral, essa atuação pode ser explicada pelo fato delas visarem a atividade de patenteamento como uma forma de geração de conhecimentos aplicados às tecnologias e colaboração (SORIA et al., 2010). Neste caso, o foco é a geração do conhecimento em si e não o lucro, limitando financeiramente a escolha das universidades quanto à proteção e o escopo geográfico de depósitos de patentes.

\footnotetext{
${ }^{5}$ Somatória elaborada desconsiderando os depósitos nos países de origem.
} 
Quadro 4 - Organizações e os seus principais mercados de interesse

\begin{tabular}{c|c|cccc}
\multicolumn{1}{c}{$\begin{array}{c}\text { País de } \\
\text { origem }\end{array}$} & Organizações globais & China & $\begin{array}{c}\text { Estados } \\
\text { Unidos }\end{array}$ & Japão & $\begin{array}{c}\text { Coréia } \\
\text { do Sul }\end{array}$ \\
\hline China & Zhejiang University & 650 & 1 & 0 & 0 \\
Japão & Iseki & 11 & 2 & 386 & 7 \\
Japão & Panasonic & 51 & 31 & 231 & 9 \\
China & Beijing University of Technology & 294 & 1 & 0 & 0 \\
China & Shanghai University & 252 & 0 & 0 & 0 \\
Japão & Yanmar & 18 & 9 & 240 & 14 \\
China & Tianjin University & 226 & 2 & 0 & 0 \\
China & Nanjin University & 225 & 0 & 0 & 0 \\
Coréia do Sul & LG & 76 & 50 & 2 & 147 \\
Alemanha & Basf & 55 & 115 & 0 & 40 \\
Fonte: Autor & & & & &
\end{tabular}

Já as empresas tiveram um número maior de depósitos em outros países, com destaque para a China e os Estados Unidos, sendo a LG a maior atuante no mercado chinês $(27,6 \%)$ e a Basf no mercado americano (54,7\%). A Yanmar, a Panasonic e a Iseki, depositaram quase a todas suas patentes em seus países de origem, porém, demonstraram um interesse atenuado na China, Coréia do Sul e Estados Unidos. Diferentemente das universidades a estratégia de patenteamento das empresas, de um modo geral, consiste na proteção das tecnologias visando o lucro e a competitividade, afim de, entre outros fatores, compensar o dispêndio financeiro gasto com depósitos.

Desde 1970, a China vem passando por diversos processos de transformações socioeconômicas, dentre os quais se destacam duas: a transição de uma economia socialista no campo para uma economia de mercado e o início de um processo de industrialização. Essas transformações vêm sendo importantes para a produção agrícola, para o aumento e melhoria dos insumos e para o crescimento da produção e produtividade. O governo chinês busca modernizar a agricultura a fim de garantir o abastecimento de commodities consideradas essenciais. Em relação aos Estados Unidos, o interesse nesse mercado se dá por ser uma potência econômica mundial que apresenta um elevado nível tecnológico em suas propriedades rurais, utilizando se de máquinas, fertilizantes, agrotóxicos, etc. Tal elevado nível tecnológico somado às contribuições geográficas, como uma grande extensão territorial e clima favorável, contribui para o aumento da produtividade. (FREITAS, [201-?]; FREITAS JÚNIOR, 2013; TOLLINE, 2013). Apesar de o Brasil ser considerado uma potência do agronegócio, nenhuma das organizações mapeadas protegeram suas tecnologias no mercado brasileiro. Este fator pode ser compreendido como uma vantagem, pois, torna tais documentos

InCID: R. Ci. Inf. e Doc., Ribeirão Preto, v. 4, n. 1, p. 107-128, jan./jun. 2013. 
de patente importantes fontes de informação para pesquisas e competidores localizados neste país.

\section{Conclusões}

Os indicadores apresentados contribuem para tomada de decisões mais racionais e direcionadas acerca de processos competitivos de organizações, governos, ou interessados em conhecer o desenvolvimento tecnológico em instrumentação agropecuária. No que tange aos resultados, a área teve um comportamento difuso em relação às principais organizações, áreas tecnológicas e uso das tecnologias. Provavelmente essa difusão foi consequência da falta de uma terminologia bem definida que culminou em uma expressão de busca relativamente abrangente.

Entre os principais titulares analisados, notou-se um interesse proveniente tanto de organizações de origem japonesa quanto chinesa no desenvolvimento dessas tecnologias. As universidades chinesas depositaram suas patentes apenas em seu país de origem, o que denota uma possível menor valoração e impacto de suas tecnologias e o interesse apenas para o âmbito nacional de atuação.

Não foram identificados depósitos de patentes no Brasil pelas organizações mapeadas, esse fato pode ter sido por um desinteresse no mercado brasileiro, ou por dificuldades com o sistema de propriedade intelectual. Contudo, as tecnologias não protegidas podem ser utilizadas por empresas brasileiras, caso tenham certeza de que a proteção não seja ampliada para o Brasil, visto que o período da análise foi bastante recente.

Em relação ao método adotado, combinar as funcionalidades da base bibliométrica Derwent Innovations Index com o software VantagePoint, para lidar com grandes volumes de dados, mostrou-se eficaz, minimizando o número de registros bibliográficos de documentos de patente processados e analisados. Contudo, análises comparativas e outros macroindicadores foram desconsiderados, o que afeta a compreensão mais ampla da área de interesse avaliada e outros insights que poderiam eventualmente surgir. Por fim, evidencia-se a necessidade de aprofundar os estudos relacionados à instrumentação agropecuária, como por exemplo, utilizar outras bases de dados, verificar novas formas e possibilidades de processamento dos dados, aprofundar a investigação sobre mercado e inovação, entre outros. Um fato relevante é que ainda há uma lacunas sobre definições e terminologias adequadas à 
temática de instrumentação agropecuária, como a elaboração de vocabulários controlados e tesauros.

\section{Referências}

ALVES, J. M. S. et al. Dinâmica inovativa no agronegócio: a inovação tecnológica na avicultura industrial por meio da análise de patentes. Cadernos de Ciência \& Tecnologia, Brasília, v. 23, n. 2/3, p. 207-233, jan. 2006.

ARAÚJO, M. Fundamentos de agronegócios. 2. ed. São Paulo: Atlas, 2007. 159 p.

BARROSO, W. et al. Patents as technological information in Latin America. World Patent Information. v. 31, n. 3, p. 207-215, sept. 2009.

BASF. Basf Brasil. 2005. Disponível em: <http://www.basf.com.br/>. Acesso em: 21 mar. 2013.

CAMPOS, I. M.; VALADARES, E. C. Inovação tecnológica e desenvolvimento econômico.[2008?]. Disponível em:

<www.schwartzman.org.br/simon/blog/inovacaomg.pdf>. Acesso em: 23 mar. 2013.

DEBACKERE, K; LUWEL, M. Patent data for monitoring S\&T portfolios. In: MOED, H. F et al.(org). Handbook of quantitative studies of science and technology: the use of publication and patent statistics in studies of S\&T Systems. Netherlands: Kluwer Academic, 2004. p. 569- 585.

DOU, $\mathrm{H}$ et al. Competitive technology assessment. Strategic patent clusters obtained with non-boolean logic. New applications of the GET command. World Patent Information, v. 12, n. 4, p. 222-229, 1990.

EMBRAPA INSTRUMENTAÇÃO AGROPECUÁRIA. IV Plano diretor da Embrapa Instrumentação Agropecuária 2008-2011-2023. São Carlos, 2008. Disponível em: $<$ http://www.cnpdia.embrapa.br/PDU_CNPDIA_2008-2011-2023.pdf>. Acesso em: 23 abr. 2013.

ERNST, H. Patent information for strategic technology management. World Patent Information, v. 25, n. 3, p. 233-242, sept. 2003.

FACULDADE DE CIÊNCIA DA INFORMAÇÃO. Glossário geral da Ciência da Informação. 2004. Disponível em:<http://www.cid.unb.br/publico/setores/100/123/sistema/m0039015.htm> Acesso em: 08 dez. 2012.

FARIA, L. I. L. Prospecção tecnológica em materiais: aumento da eficiência do tratamento bibliométrico. Aplicação na análise de tratamentos de superfícies resistentes ao desgaste. 2001. 187 f. Tese (Doutorado em Ciência e Engenharia de Materiais) - Universidade Federal de São Carlos, São Carlos, 2001. 
FERREIRA, A. A. et al. Patente como instrumento competitivo e como fonte de informação tecnológica. Gestão \& Produção, São Carlos, v. 16, n. 2, p. 209-221, jun. 2009.

FREITAS, E. Agropecuária dos Estados Unidos. [201-?]. Disponível em:

$<$ http://www.mundoeducacao.com.br/geografia/agropecuaria-dos-estados-unidos.htm>. Acesso em: 20 mar. 2013.

FREITAS JÚNIOR, G. China também da um salto na agricultura. Valor Econômico, São Paulo, 05. mar. 2013. Disponível em:

$<$ http://clippingmp.planejamento.gov.br/cadastros/noticias/2013/3/5/china-tambem-da-umsalto-na-agricultura>. Acesso em: 20 mar. 2013.

FUNDAÇÃO NACIONAL DA QUALIDADE. Cadernos de Excelência: informações e conhecimento. São Paulo: Fundação Nacional da Qualidade, 2008. 21 p. (Cadernos de Excelência, n. 5)

HU, A. G.; JEFFERSON, G. H. A great wall of patents: what is behind China's recent patent explosion?. Journal of Development Economics, v. 90, n.1, p.57-68, jan. 2009

INSTITUTO NACIONAL DA PROPRIEDADE INDUSTRIAL. Portal INPI. [2012?].

Disponível em: <http://www.inpi.gov.br/portal/> Acesso em 08 jan. 2013.

INSTRUMENT SOCIETY OF AMERICAN; AMERICAN NATIONAL STANDARDS INSTITUTE. Process instrumentation terminology. Durham: Instrument Society of America, 1979. 66 p.

ISEKI. Iseki \& Co Ltda. [entre 1997 e 2013]. Disponível em:

$<$ http://www.iseki.co.jp/english/index.html>. Acesso em: 20 mar. 2013.

LG ELECTRONICS. Lg: life's good. 2012. Disponível em:

$<$ http://www.lg.com/global/products/>. Acesso em: 22 mar. 2013.

MARCO, S. A. Inteligência competitiva: definições e contextualização. Transinformação, Campinas, v. 11, n. 2, p. 95-102, maio/ago. 1999.

MARTIN, A. D.; FARIA, L. I. L. Monitoramento de patentes sobre plásticos biodegradáveis. In: WORKSHOP BRASILEIRO DE INTELIGÊNCIA COMPETITIVA E GESTÃO DO CONHECIMENTO, 3., 2002, São Paulo Anais...São Paulo, 2002.

MILANEZ, D. H. Nanotecnologia: indicadores tecnológicos sobre os avanços em materiais a partir da análise de documentos de patentes. 2011. 176 f. Dissertação (Mestrado em Ciência e Engenharia dos Materiais) - Centro de Ciências Exatas e de Tecnologia, Universidade Federal de São Carlos, São Carlos, 2011

NASSAR, A. M; NOGUEIRA. S. China. Agroanalysis FGV. 2007. Disponível em: $<$ http://www.agroanalysis.com.br/especiais_detalhe.php?idEspecial=16> Acesso em: 10 mar. 2013. 
OBSERVATOIRE DES SCIENCES ET DES TECHNIQUES. Indicateurs de sciences et de technologies. Paris: Economica, 2008. Disponível em: <http://www.obsost.fr/fileadmin/medias/PDF/R08_Complet_Liens.pdf>. Acesso em: 19 fev. 2012.

ORGANIZAÇÃO MUNDIAL DA PROPRIEDADE INTELECTUAL. Guide to use patent information. Genebra: Organização Mundial da Propriedade Intelectual, 2012 Disponível em: < http://www.wipo.int/freepublications/en/patents/434/wipo_pub_1434_03.pdf> Acesso em: 8 fev. 2013.

ORGANIZAÇÃO PARA COOPERAÇÃO E DESENVOLVIMENTO ECONÔMICO. Manual de Oslo: diretrizes para coleta e interpretação de dados sobre inovação. 3 ed. Eurostat; FINEP. 2005. 163 p.

ORGANIZAÇÃO PARA COOPERAÇÃO E DESENVOLVIMENTO ECONÔMICO. OECD patent statistics manual. Paris: Organização para Cooperação e Desenvolvimento Econômico, 2009. 158 p.

OCTAVIANO, V. L. Avaliação da terminologia utilizada em instrumentação agropecuária. Ciência da informação, v. 24, n. 3, p. 1-10, set./dez. 1995.

OKUBO, Y. Bibliometric indicators and analysis of research systems: methods and examples. Paris: Organização para Cooperação e Desenvolvimento Econômico, 1997. 70 p.

PANASONIC CORPORATION. Panasonic globeride. 2013. Disponível em:< http://www.panasonic.net/> Acesso em: 22 mar. 2013.

PEREIRA, S.; PAULA, R. M. A inovação tecnológica como ferramenta competitiva no agronegócio. Revista Univap, São José dos Campos, v. 13, n. 24, p. 1-6, out. 2006

PERET, C. M. et al. Patent generation and the technological development of refractories and steelmaking. Refractories Applications \& News, Rolla, v. 12, n. 1, p. 10-14, 2007.

PORTAL BRASIL. Economia: agronegócio. 2010. Disponível em:

<http://www.brasil.gov.br/sobre/economia/setores-da-economia/agronegocio> Acesso em: 20 mar. 2013>

PORTER, A. L.; NEWMAN, N. C. Patent Profiling for competitive advantage. In: MOED, H. F et al.(org). Handbook of quantitative studies of science and technology: the use of publication and patent statistics in studies of S\&T Systems. Netherlands: Kluwer Academic, 2004. p. 587- 612

QUONIAM, L. et al. Bibliometric analysis of patent documents for R\&D. Research Evaluation, Oxford, v. 3, n. 1, p. 13-18, 1993.

RAMOS, R. C. Elaboração de indicadores de patentes sobre nanotecnologia aplicada ao agronegócio. 2012. 112 f. Dissertação (Mestrado em Ciência Tecnologia e Sociedade), Programa de Pós-graduação Ciência Tecnologia e Sociedade, Universidade Federal de São Carlos, São Carlos, 2012.

RIBEIRO, M. A. Instrumentação. 8. ed. Salvador: Marco Antônio Ribeiro, 2007. 329 p. 
RUFINO, José Luís dos Santos. Origem e conceito do agronegócio. Informe Agropecuário, Belo Horizonte, v. 20, n Q 199, p. 17-19, jul. /ago. 1999

SAUER, S. Reforma agrária e geração de emprego e renda no meio rural. São Paulo: ABET, 1998. 127 p.

SORIA, A. F. et al. Geração de patentes em universidades: um estudo exploratório. Revista de Administração Faces Journal, Belo Horizonte, v. 9, n. 3, p. 95-116, jul./set. 2010.

THOMSON. Derwent innovations index: tools of the trade. London: Thomson Scientific, 2007. 114 p.

TOLLINE, H. A China e a segurança alimentar. Correio Braziliense, Brasília, 14 jan. 2013. Disponível em:

$<$ https://conteudoclippingmp.planejamento.gov.br/cadastros/noticias/2013/1/14/a-china-e-aseguranca-alimentar>. Acesso em: 20 mar. 2013.

VANTAGEPOINT. Products. 2013. Disponível em:

<http://www.thevantagepoint.com/products.html> Acesso em: 08 fev. 2013.

VIAN, C. E. F.; ANDRADE JÚNIOR, A. M. A evolução histórica da indústria de máquinas agrícolas no mundo. In: CONGRESSO SOCIEDADE BRASILEIRA DE ECONOMIA ADMINISTRAÇÃO E SOCIOLOGIA RURAL, 48., 2010, Campo Grande. Anais... Campo Grande: SOBER, 2010. p. 1-19.

WATTS, R. J.; PORTER, A. L. Innovation forecasting. Technological Forecasting and Social Change, v. 56, n.1, p. 25-47, set. 1997.

YANMAR. Yanmar: solutioneering together. 2010. Disponível em: <http://www.yanmar.co.jp/en/>. Acesso em: 21 mar. 2013. 